\title{
Delimitação das classes de capacidade de suo das terras da microbacia do Córrego Três Barras - Botucatu (SP)
}

\author{
Sérgio Campos ${ }^{1}$ \\ Muriel Cicatti Emanoeli Soares ${ }^{1}$ \\ Raquel Cavasini ${ }^{1}$ \\ Teresa Cristina Tarlé Pissarra ${ }^{2}$ \\ Flávia Mazzer Rodrigues ${ }^{2}$ \\ ${ }^{1}$ Faculdade de Ciências Agronômicas/UNESP \\ Rua José Barbosa de Barros, 1780 \\ 18610 - 307 - Botucatu - SP, Brasil \\ seca@fca.unesp.br \\ ${ }^{2}$ Faculdade de Ciências Agrárias/UNESP \\ Rodovia Donato Castellane, $\mathrm{s} / \mathrm{n}$ \\ 14884 - 900 - Jaboticabal - SP, Brasil \\ teresap@fcav.unesp.br
}

Resumo: O trabalho objetivou analisar as classes de capacidade de uso das terras da Microbacia Córrego Três Barras - Botucatu (SP). A área situa-se entre as coordenadas geográficas: latitude $22^{\circ} 47^{\prime} 08^{\prime \prime}$ a $22^{\circ} 51^{\prime} 29^{\prime \prime} \mathrm{S}$ e longitudes $48^{\circ} 22^{\prime} 16^{\prime \prime}$ a $48^{\circ} 26^{\prime} 12^{\prime \prime} \mathrm{W}$ Gr., apresentando uma área de 1651 ha. As classes de capacidade de uso da área foram obtidas através do sistema de classificação constante do manual para Levantamento Utilitário do Meio Físico e Classificação de Terras no Sistema de Capacidade de Uso. As classes de capacidade de uso do solo III e IV formadas pelas subclasses IIIs, IIIs,e, IVe, IVs e IVs,e, ocuparam 72,03\% da área, mostrando o grande potencial de uso para culturas anuais, perenes, pastagens e ou reflorestamentos. A subclasse IVs foi a mais significativa (45\%) da área.

Palavras Chaves: classes de capacidade de uso, geoprocessamento.

\begin{abstract}
The work aimed at to analyze the use capability use of Stream Três Barras watershed - Botucatu (SP). The area locates among the geographical coordinates: latitude $22^{\circ} 47^{\prime} 08^{\prime \prime}$ to $22^{\circ} 51^{\prime} 29^{\prime \prime} \mathrm{S}$ and longitudes $48^{\circ} 22^{\prime} 16^{\prime \prime}$ to $48^{\circ} 26^{\prime} 12^{\prime \prime} \mathrm{W}$ Gr., presenting an area of 1651 ha. The use capacity of classes of the area were obtained through the system of constant classification of the manual for Utilitarian Rising of the Physical Middle and Classification of Lands in the Use Capability Classes. The use capability classes of the soil III and IV formed by the subclasses IIIs, IIIs,e, IVe, IVs and IVs,e, occupied $72,03 \%$ of the area, showing the great use potential for cultures annual, perennial, pastures and or reforestations. The subclass IVs was the most significant (45\%) of the area.
\end{abstract}

Key words: use capability classes, geoprocessing

\section{Introdução}

Sendo a agricultura uma das principais formas de exploração das terras deste país, é de grande valia o levantamento das condições do solo, meio natural básico para a atividade agropecuária, praticando um modo exploratório de forma consciente baseado na adequação de práticas conservacionistas a dados tipos de solo, tirando melhor proveito e aumentando a longevidade, possibilitando o aumento da potencialidade e a utilização pelas gerações futuras.

É de extrema importância a interpretação dos levantamentos de solo, visto que a utilização deste recurso natural muitas vezes está sendo realizada de maneira errônea limitando o uso. Para o levantamento é feita a classificação das terras de acordo com a sua capacidade de uso, condicionada por manejos diferenciados, que quando agrupadas em classes permite a utilização de técnicas específicas. 
A partir de limitações demarcadas pela capacidade de uso decide-se em optar quanto à realização de alguma infra-estrutura ou benfeitoria interna ou externamente a propriedade, abrangendo somente aquela localidade ou tomando medidas que considere um espaço mais amplo para real solução de algum problema relacionado com mais de uma propriedade ou até mesmo com a bacia como um todo.

A capacidade de uso visa o aproveitamento das condições do solo com um mínimo de perdas, baseando-se num detalhamento expressivo dos fatores que possam influenciar a estruturação e composição deste meio, tais como relevo, erosão, solo, clima, entre outros; tornando-se mais confiáveis as bases para planejamento de uso racional.

O presente trabalho de pesquisa tem por objetivo delimitar as classes de capacidade de uso das terras da Microbacia Córrego Três Barras - Botucatu (SP), realizando para isso, a elaboração da carta de solos e a tomada de medidas na carta de declive.

\section{Material e Métodos}

O presente trabalho foi desenvolvido na microbacia do Córrego Três Barras - Botucatu (SP), situada geograficamente entre as coordenadas geográficas: latitude $22^{\circ} 47^{\prime} 08^{\prime \prime}$ a $22^{\circ} 51^{\prime} 29^{\prime \prime} \mathrm{S}$ e longitudes $48^{\circ} 22^{\prime} 16^{\prime \prime}$ a $48^{\circ} 26^{\prime} 12^{\prime \prime}$ W Gr., apresentando uma área de 1656 ha.

Neste trabalho, os pontos de controle no processo de georreferenciamento da carta clinográfica, foram extraídos das cartas planialtimétricas do Instituto Brasileiro de Geografia e Estatística - IBGE, em escala 1: 50.000, referente à folha de Botucatu (SF-22-R-IV-3), escala 1:50000.

Extraiu-se também das cartas citadas a demarcação manual do limite da microbacia as curvas de nível eqüidistantes de 20 em 20 metros para a confecção do ábaco das declividades.

No georreferenciamento, utilizaram-se dois arquivos de pontos de controle, conforme sugerido por Eastman (1998) para chegar-se a um erro médio quadrático abaixo da metade da resolução desejada, sendo o primeiro da imagem digital e o outro, da carta topográfica de Botucatu. Foram determinadas as coordenadas de cada ponto e com estes dados foi feito um arquivo de correspondência, através do comando "Edit" do menu "Database Query", presente no módulo "Analysis".

$\mathrm{Na}$ próxima seguinte, houve a delimitação da imagem, extraindo-se apenas a área da microbacia do Córrego Três Barras, sendo posteriormente demarcadas as áreas de treinamento sobre a imagem com o cursor e o mouse. Essas áreas foram demarcadas sobre grande número de locais, buscando-se abranger todas as variações de cada ocupação do solo.

Em seguida realizou-se a digitalização do limite da microbacia e também a digitalização das classes de declive e unidades de solo, sendo atribuído a cada classe e a cada unidade um dado identificador correspondente. A digitalização foi feita através do "software" CARTALINX.

Para a confecção da carta de solos referente à área da Microbacia Córrego Três Barras Botucatu (SP), utilizou-se dos dados do Mapa Pedológico do Estado de São Paulo (Oliveira et al., 1999), em escala 1:500.000.

Posteriormente realizou-se a scannerização da área e sua importação para o software IDRISI versão 3.2 pelo módulo File/ Import num formato passível de entrada, sendo posteriormente georreferenciada.

Para a obtenção da Carta Clinográfica utilizou do ábaco das declividades elaborado conforme De Biasi (1970), realizando inicialmente a transferência direta das isolinhas das cartas planialtimétricas, representativas da Microbacia Córrego Três Barras - Botucatu (SP), editadas pelo Instituto Brasileiro de Geografia e Estatística - IBGE, em escala 1: 50.000, referente à folha de Botucatu (SF-22-R-IV-3), demarcando-se também pontos de controle. 
As classes de capacidade de uso da área de estudo foram determinadas de acordo com o sistema de classificação constante do Manual para Levantamento Utilitário do Meio Físico e Classificação de Terras de Capacidade de Uso. Para a classificação, seguiram-se os critérios constantes do quadro de julgamento confeccionado conforme França (1963) e Lepsch et al. (1991) e, de acordo com adaptação regional compilada por Zimback \& Rodrigues (1993).

\section{Resultados e Discussão}

Após a elaboração do levantamento de solos e das classes de declive, passou-se à execução do levantamento das classes de capacidade de uso da terra da área em estudo. O procedimento de classificação das classes foi baseado no cruzamento entres as classes de solo, com suas diferentes declividades. Em seguida, avaliaram-se os demais parâmetros através da tabela de julgamento até chegar-se à classe de capacidade de uso da terra (Tabela 1).

Tabela1. Determinação das classes de capacidade de uso pelos critérios da tabela de julgamento:

\begin{tabular}{|c|c|c|c|c|c|c|c|c|c|c|c|c|}
\hline Áreas & F.A. & P.E. & P.D. & Def. & Ped. & R.I. & Dec. & E.L. & E.S. & V. & Classe & Subclasse \\
\hline $\mathrm{LVd} / 3-6$ & III & $\mathrm{I}$ & $\mathrm{I}$ & $\mathrm{I}$ & $\mathrm{I}$ & I & II & II & II & $\mathrm{I}$ & III & IIIs \\
\hline LVd/6-12 & III & I & I & II & I & I & III & II & II & I & III & IIIs,e \\
\hline LVd/12-20 & III & I & I & II & I & I & IV & II & II & I & IV & IVe \\
\hline$L V d / 20-40$ & III & I & I & III & I & I & VI & II & II & I & VI & VIe \\
\hline LVAd1/0-3 & III & I & I & I & I & I & I & II & II & I & III & IIIs \\
\hline LVAd1/3-6 & III & I & I & I & I & I & II & II & II & I & III & IIIs \\
\hline LVAd1/6-12 & III & I & I & II & I & I & III & II & II & $\mathrm{I}$ & III & IIIs,e \\
\hline LVAd1/12-20 & III & I & I & II & I & I & IV & II & II & I & IV & IVe \\
\hline LVAd1/20-40 & III & I & I & III & I & I & VI & II & II & $\mathrm{I}$ & VI & VIe \\
\hline NVdf/6-12 & III & I & II & I & I & I & III & I & II & $\mathrm{I}$ & III & IIIs,e \\
\hline NVdf/12-20 & III & I & II & I & I & I & IV & I & II & $\mathrm{I}$ & IV & IVe \\
\hline NVdf/20-40 & III & I & II & II & I & I & VI & I & II & $\mathrm{I}$ & VI & VIe \\
\hline $\mathbf{N V d f} />40$ & III & I & II & II & I & I & VII & I & II & I & VII & VIIe \\
\hline GXbd/0-3 & III & I & V & II & I & V & I & II & II & I & V & Va \\
\hline GXbd/3-6 & III & I & V & III & I & V & II & II & II & I & V & Va \\
\hline GXbd/6-12 & III & I & V & III & I & III & III & II & II & $\mathrm{I}$ & V & Va \\
\hline GXbd/12-20 & III & I & V & IV & I & III & IV & II & II & I & V & Va \\
\hline RLe/0-3 & II & II & III & II & IV & I & I & II & II & $\mathrm{I}$ & IV & IVs \\
\hline RLe/3-6 & II & II & III & II & IV & I & II & II & II & $\mathrm{I}$ & IV & IVs \\
\hline RLe/6-12 & II & II & III & III & IV & I & III & II & II & $\mathrm{I}$ & IV & IVs \\
\hline RLe/12-20 & II & II & III & III & IV & I & IV & II & II & I & IV & IVs,e \\
\hline RLe/20-40 & II & II & III & IV & IV & I & VI & II & II & $\mathrm{I}$ & VI & VIe \\
\hline RLe/>40 & II & II & III & IV & IV & I & VII & II & II & I & VII & VIIe \\
\hline
\end{tabular}

F.A. - Fertilidade aparente; P.E. - Profundidade efetiva; P.D. - Permeabilidade e drenagem interna; Def Deflúvio; Ped - Pedregosidade; R.I. - Risco de inundação; Dec - Declividade; E.L. - Erosão laminar;

E.S. - Erosão em sulcos; V - Voçorocas.

Posteriormente, reclassificaram-se através do módulo reclass do Idrisi, todas as áreas com as mesmas características, juntando-as em um mesmo identificador, obtendo-se desta forma o mapa de capacidade de uso das terras da microbacia Córrego Três Barras no município de Botucatu/SP (Figura 1 e Tabela 2). 


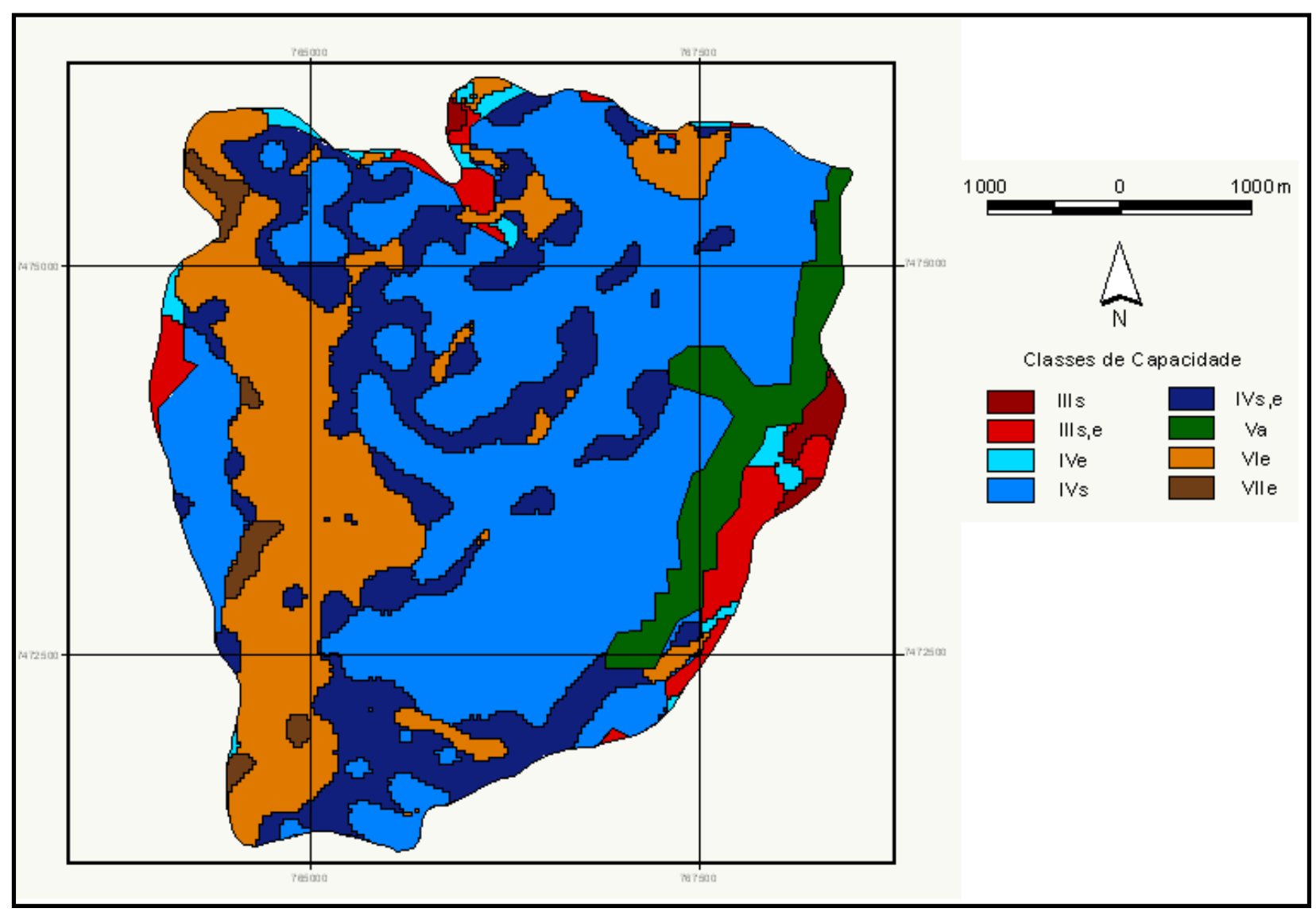

Figura 1. Subclasses de capacidade de uso da terra na microbacia Córrego Três Barras Botucatu (SP).

Tabela 2. Subclasses de capacidade de uso da terra na microbacia do Córrego Três Barras Botucatu (SP).

\begin{tabular}{ccc}
\hline Classes de capacidade & Área total (ha) & Porcentagem da área total (\%) \\
\hline IIIs & 14,88 & 0,90 \\
IIIs,e & 58,40 & 3,54 \\
IVe & 24,10 & 1,46 \\
IVs & 771,57 & 46,73 \\
IVs,e & 320,29 & 19,40 \\
VIIe & 23,12 & 1,40 \\
VIe & 340,32 & 20,61 \\
Va & 98,32 & 5,96 \\
\hline \hline
\end{tabular}

De acordo com Lepsch et al. (1991), são próprias para utilização com culturas anuais, perenes, pastagens e ou reflorestamentos e vida silvestre as terras das classes III e IV, atentandose para cada classe e subclasse, representando $72,03 \%(1189,24 \mathrm{ha})$ da área total da microbacia. Ainda conforme este autor, são consideradas terras impróprias para cultivos intensivos, mas ainda adaptadas para pastagens ou reflorestamento e ou vida silvestre as terras das classes V, VI e VII, representando $27,97 \%$ (461,76ha) da área total da microbacia. 


\section{Conclusões}

Os resultados permitiram inferir que a classe de capacidade III e IV, divididas na nas subclasses IIIs, IIIs,e, IVe, IVs e IVs,e, ocuparam 72,03\% da área total da microbacia mostrando o grande potencial de uso para culturas anuais, perenes, pastagens e ou reflorestamentos, sendo a sub-classe IVs a mais significativa, pois ocupa mais de $45 \%$ da área da microbacia.

\section{Referências Bibliográficas}

Oliveira, J.B de. Solos do Estado de São Paulo: descrição das classes registradas no mapa pedológico. Campinas: Instituto Agronômico de Campinas, 1999. 108p.

França, G. V. A classificação de terras de acordo com sua capacidade de uso com base para um programa de conservação do solo. In: Congresso Nacional da Conservação do Solo, 1., 1963, Campinas. Anais... Campinas Secretaria da Agricultura, DEMA, 1963. p.339-408.

Lepsch, I. F., Bellinazi Jr., R., Bertolini, D., Espíndola, C. R. Manual para levantamento utilitário do meio físico e classificação de terras no sistema de capacidade de uso. Campinas: Sociedade Brasileira de Ciência do Solo, 1991. 175p.

Eastman, J. R. Idrisi for windows - Manual do Usuário: Introdução e Exercícios Tutoriais. Editores da versão em português, Heinrich Hasenack e Eliseu Weber. Porto Alegre, UFRGS Centro de Recursos do Idrisi, 1998. 240 p.

Zimback, C. R. L., Rodrigues, R. M. Determinação da capacidade de uso das terras da Fazenda São Manuel UNESP. UNESP, Botucatu, 1993. 36p. 